\title{
Centralised Optical Monitoring of Tree-structured Passive Optical Networks using a Raman-assisted OTDR
}

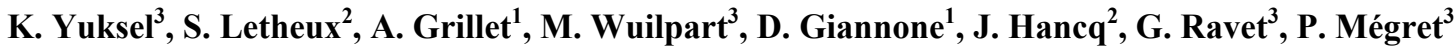 \\ ${ }^{1}$ Multitel, Rue Pierre et Marie Curie 2, 7000 Mons, Belgium \\ ${ }^{2}$ Faculté Polytechnique de Mons, TCTS lab, Bd Dolez 31, 7000 Mons, Belgium \\ ${ }^{3}$ Faculté Polytechnique de Mons, SET, Bd Dolez 31, 7000 Mons, Belgium \\ Tel:+32 (0)6534 2829, Fax:+32 (0)65342799, e-mail: grillet@multitel.be
}

\begin{abstract}
Monitoring of tree-structured Passive Optical Networks from a central office is difficult to achieve using a standard OTDR. Switchable reflective elements placed at the ONU can help to discriminate between the branches. In parallel, a Raman assisted OTDR is shown to increase the dynamic range of the reflectometry measurement, thus enabling to detect more faults in the network, and especially fibre breaks after the splitter.
\end{abstract}

Keywords: OTDR, PON, Raman, monitoring.

\section{INTRODUCTION}

FTTx networks such as Passive Optical Networks (PON) are progressively becoming reality while commercial deployments are reported worldwide [1]. Operating these networks however requires adequate means for a costeffective monitoring. Especially for troubleshooting faults in the network, the possibility to remotely differentiate between an equipment failure and a fibre break, and subsequently to localise it in the network, is of great importance for operators. Remote monitoring of fibre networks via Optical Time Domain Reflectometry (OTDR) techniques is widely used in point-to-point topologies but has major limitations in tree-structured PONs, where all backscattered traces add together at the Optical Line Terminal (OLT) location, thereby making it difficult to differentiate between the branches [2].

Several techniques were proposed in the past to overcome this limit. In particular, the possibility to make upstream OTDR measurements from the Optical Network Unit (ONU) using the existing transmitter has been explored [3]. However, this technique has the disadvantage of not being centralised, and in addition would loose its utility in case of a fibre break. Therefore, a centralised solution should be preferred. Usage of a multiwavelength OTDR to observe the branches separately was reported [4] but this solution is not viable due to the high cost of a tuneable OTDR. Another simple and effective way is to introduce a reference reflection at the end of each branch and to detect the presence and height variation of reference reflection peaks [5]. However, the requirement of having branches of different lengths makes it difficult for operators to be a practical solution. An alternative was then proposed consisting in placing at each ONU location a switchable reflective element (SRE), whose signature on the OTDR trace would identify the branch being monitored [6]. The system is shown on Fig. 1.

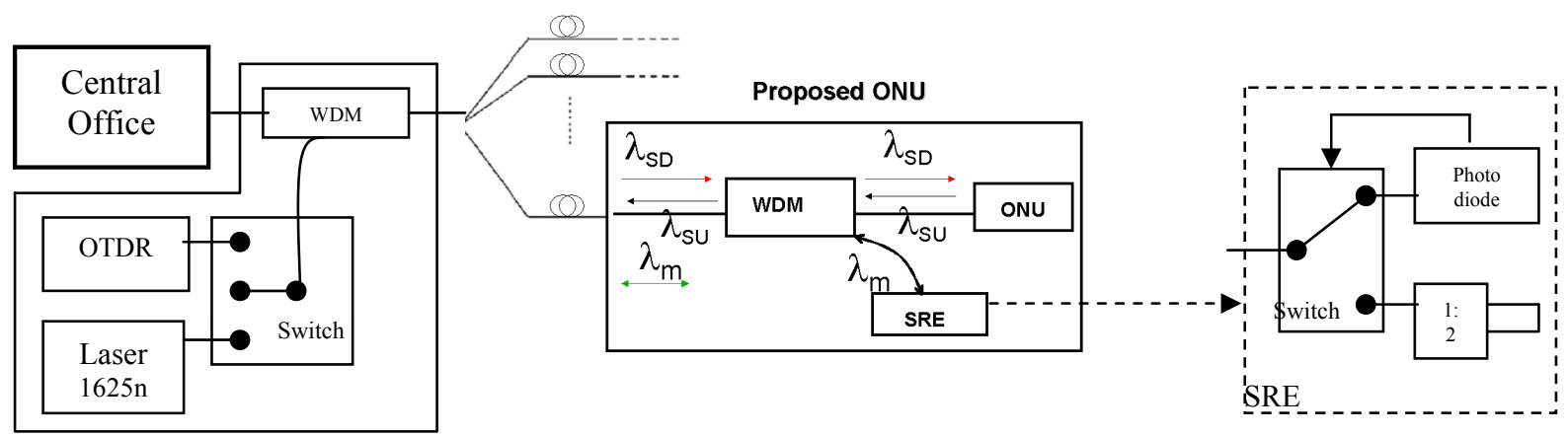

Fig. 1. Principle of the centralised method for in-service monitoring, with a schematic description of the SRE (right).

It operates at $1625 \mathrm{~nm}$ and therefore has no impact on the data traffic. The SRE is colorless device and its design is compatible with planar technologies, and thus mass production. It consists of an optical switch with a photodiode on one branch (non-reflective state; default state) and a mirror on the other branch (reflective state), the position of the switch being controlled by the photodiode. Therefore, each SRE can be remotely switched from the Central Office (CO) by sending a downstream coded signal through the transmission line at $1625 \mathrm{~nm}$. If the code received at the photodiode corresponds to the ONU address, the switch will be actuated and the SRE will turn to the reflective state. In the mean time, all other SREs will remain in the non-reflective position. During the monitoring, the SREs are thus successively remotely switched on and off from the, CO and consequently there is only one peak at a time on the OTDR trace. The system is therefore capable of localising 
and quantifying several faults successively in the same branch or in different branches, even if they occur at the same distance $[7,8]$. The resolution in terms of fault detection and quantification depends on the dynamic range (DR) of the OTDR, the splitting ratio of the PON and the attenuation in each branch.

In the past years however, the splitting ratio of installed PON has moved to higher values. The sensitivity in terms of fault detection via a centralised OTDR is consequently significantly degraded after the splitter, since OTDRs have a limited DR with respect to a given spatial resolution. As a result, less faults can then be detected, at the expense of the solution efficiency. In this paper, we report on a technique allowing to increase the dynamic range of the OTDR from the CO only. The solution is based on Raman amplification and was initially demonstrated to make distributed Raman gain measurement in installed fibres [9]. We implemented this technique in our PON test bed. We measured a $16 \mathrm{~dB}$ (return) gain in the dynamic range of the OTDR, which enabled us to detect more faults in the network.

\section{EXPERIMENTAL SET-UP}

Raman amplification is a well known and powerful amplification technique that allows to increase the reach or capacity of installed fibre links from the line terminal, with low noise impact on the propagating channels. Similarly to signal pulses, a propagating pump can therefore amplify the OTDR pulses propagating in the fibre. We implemented a CW pump (Raman laser) on our test bed at the OLT location using a WDM multiplexer as shown on Fig. 2. The pump laser wavelength is chosen so that the OTDR wavelength lies within the corresponding Raman gain bandwidth. The PON is further equipped with interference filters allowing to suppress as much as possible the amount of backscattering light at the Raman laser wavelength. Besides, because the Raman laser is also generating spontaneous Raman noise and thus broadband backscattered signal at the OTDR wavelength, we implemented a narrow band-pass filter at the reception. The amount of CW noise is then significantly reduced, however the same applies to the OTDR backscattered signal. Therefore, we used an amplified narrowband laser beam to generate the interrogating pulses instead of the wideband laser beam from the OTDR. The external laser source (1550 nm in order to be within the Raman gain bandwidth of our pump)is externally driven with the OTDR pulses via a photodiode, a pulse generator and an acousto-optic modulator, and lies within the bandwidth of the narrow band filter. The power of the new OTDR pulses being launched into the PON after amplification (EDFA)was measured at $11 \mathrm{dBm}$ (peak power).

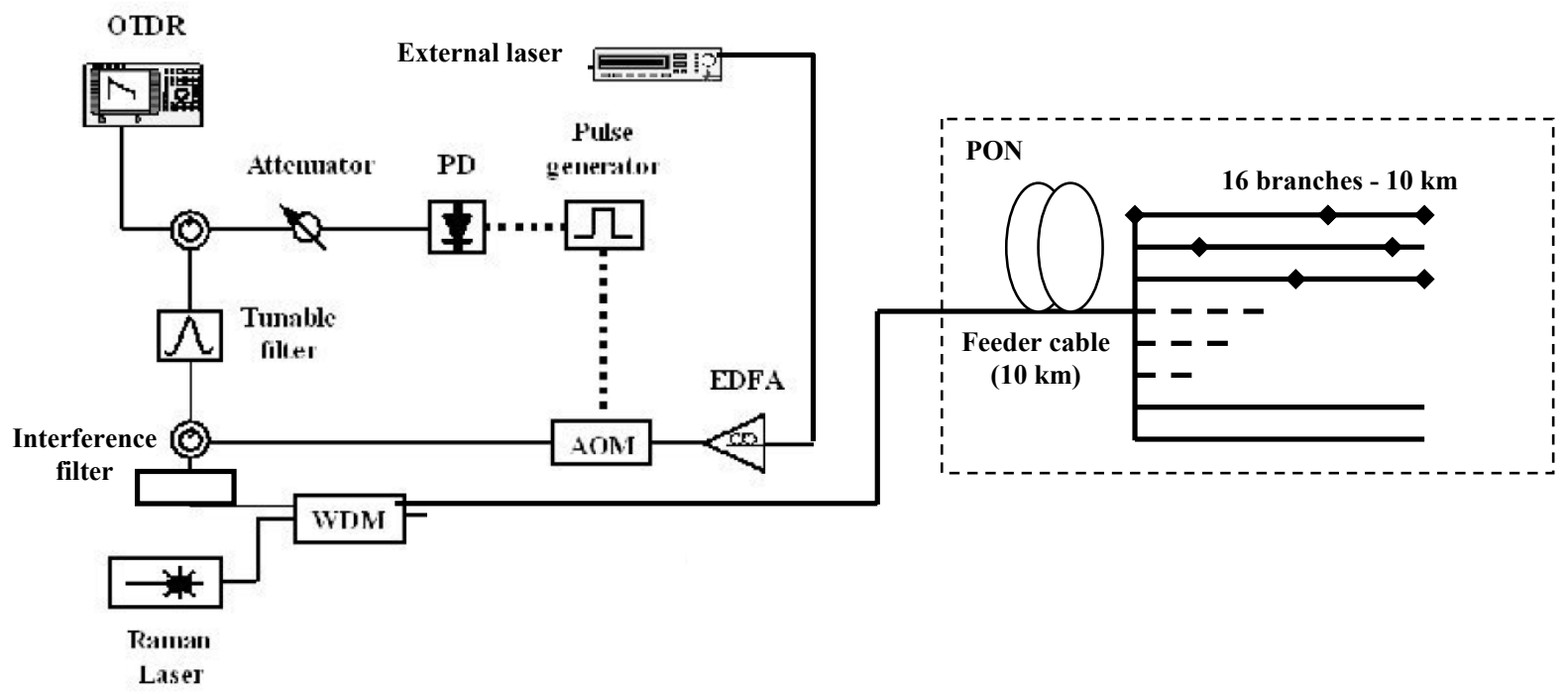

Figure 2. Experimental set-up of the Raman assisted OTDR. The Raman pump wavelength is $1455 \mathrm{~nm}$, the OTDR and external laser wavelengths are $1550 \mathrm{~nm}$.

\section{RESULTS}

The test-bed used is a PON with 1:16 splitter as shown on Fig. 2. The branches are split into several sections of fibre, so that faults can easily be inserted in the network using attenuators, damaged connectors or even fibre breaks. The PON is fully compatible with ITU-T Classes A to C for GPON.

We first assessed our technique by interrogating the PON test bed with no faults. In this experiment, the SREs were not used at the end of the branches, and consequently there is no end reflection peak on the OTDR traces. The pump power was varied from 0 to $1.5 \mathrm{~W}$, and it was found that $870 \mathrm{~mW}$ was necessary to observe amplification. Fig. 3 clearly shows an increase in the amount of backscattered light, with a corresponding gain in 
the dynamic range of $16 \mathrm{~dB}$ (return gain) after the splitter. The enhanced DR allows having a higher signal reflected or backscattered to the OTDR detector, and therefore a better signal to noise ratio, which in turns eases the fault detection and location algorithm. This is illustrated in Fig. 4, which compares the OTDR trace taken at a distance of $16 \mathrm{~km}$ from the Central Office in both configurations (Raman pump on and off). One can see that the amplitude of the noise has been dramatically reduced, thereby allowing to detect more events. In the first case (Fig. 4), it was a fibre break on branch \#3. In a second experiment (fig. 5), we repaired the fibre break, but with a pair of damaged FC/PC connectors. it was still impossible to detect the fault (FC/PC connector) at a first check, because its associated return loss was lower than the noise on the OTDR trace. The Raman laser was then turned on, and the fault could be detected with a pump power of $1.5 \mathrm{~W}$.

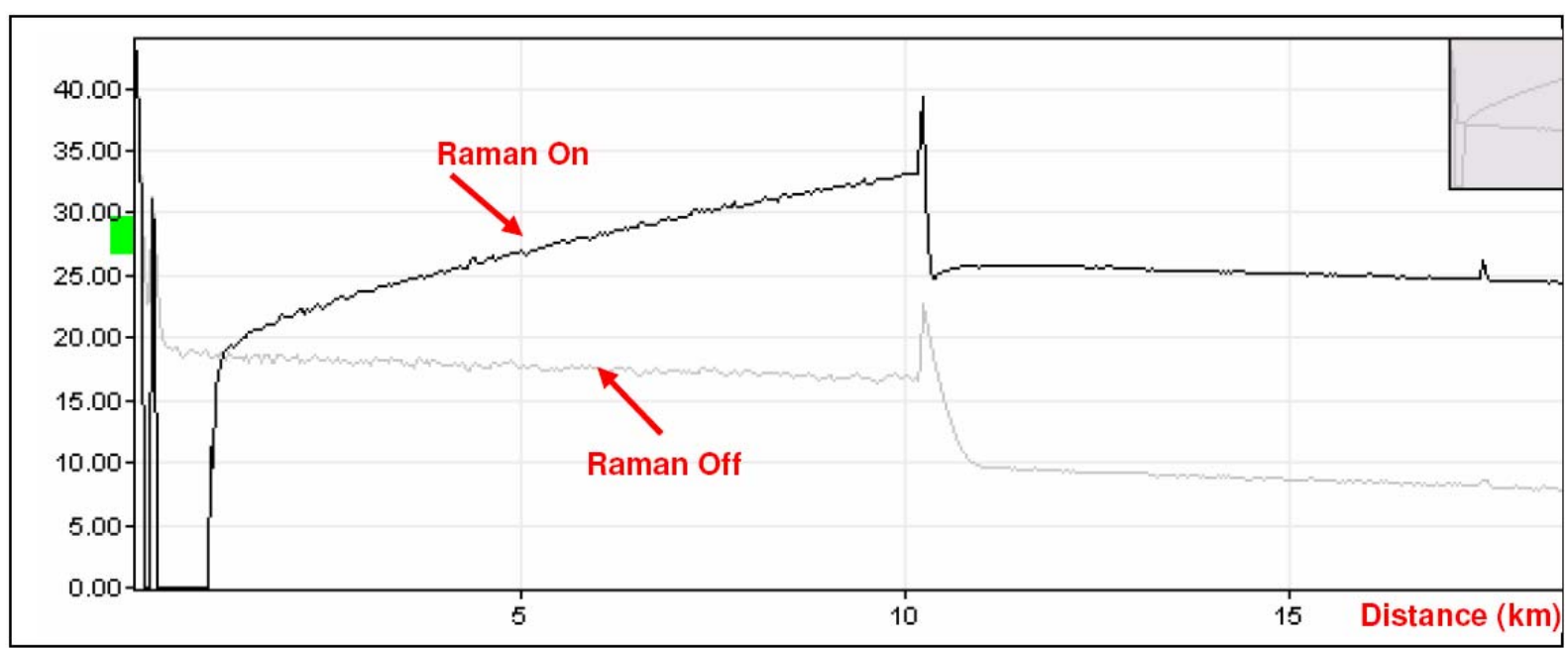

Figure 3. OTDR trace with and without Raman pump in a 1:16 PON architecture.
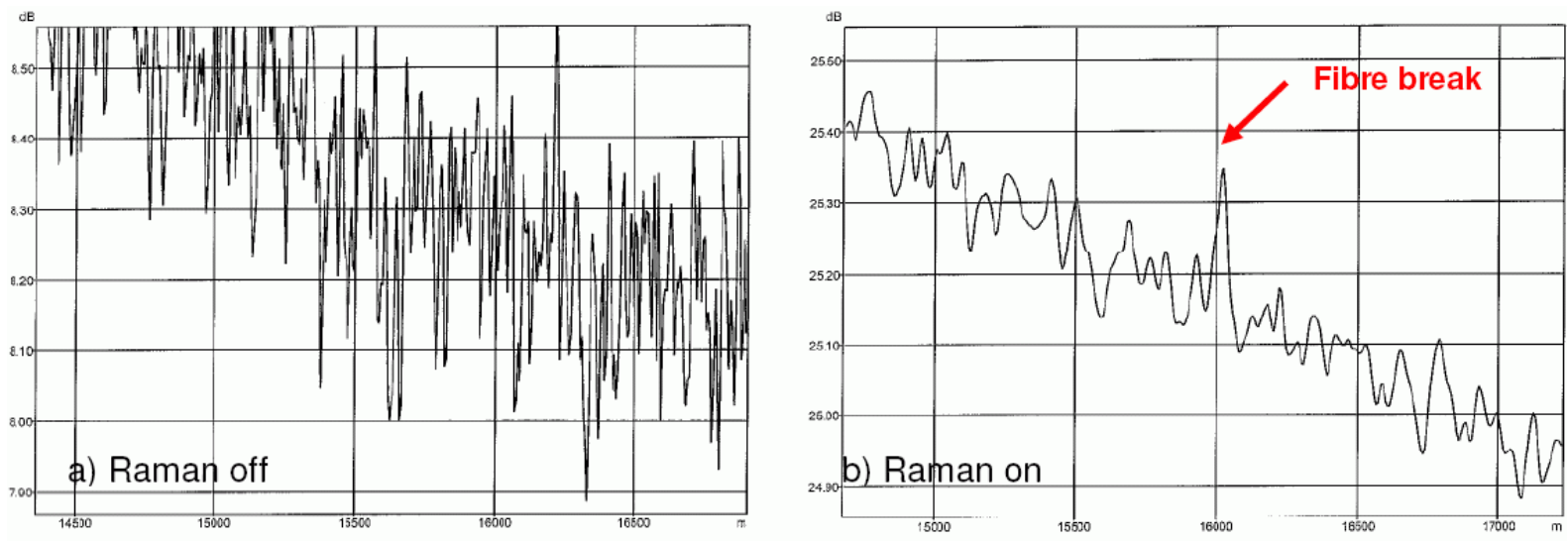

Figure 4. Zoom of the previous OTDR traces at $16 \mathrm{~km}$ from the CO. (a) (standard conditions), the noise is too important. (b) (Raman pump on), the amplitude of the noise has been significantly reduced and faults can now be detected.

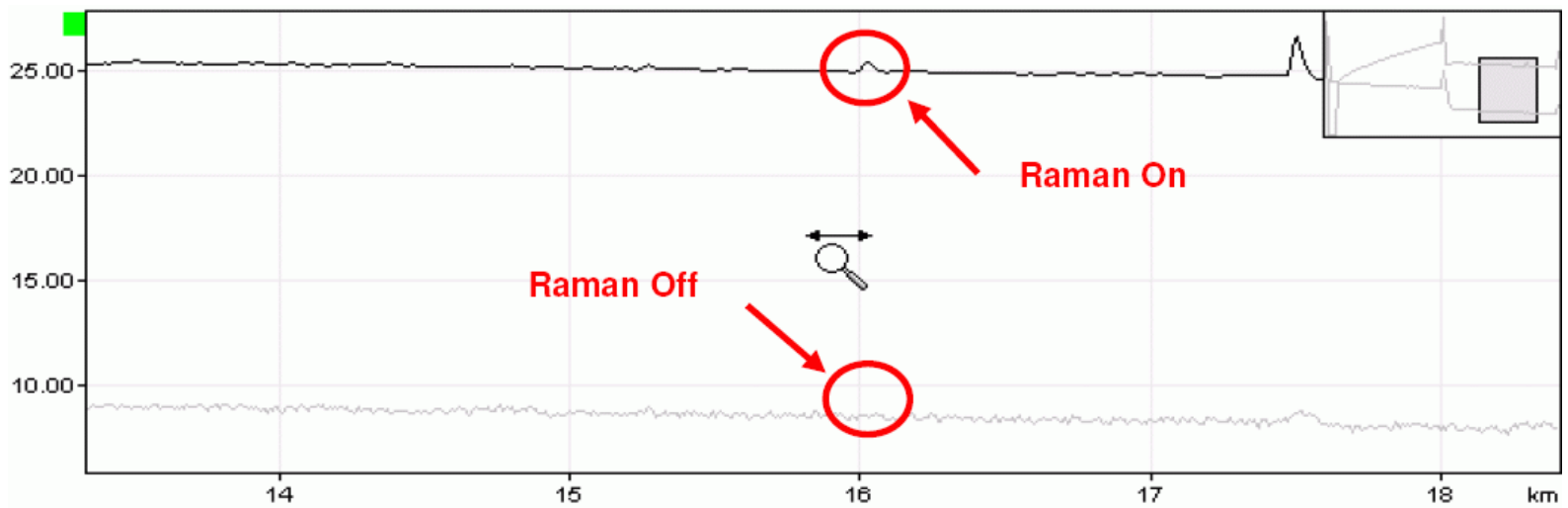

Figure 5. Fault location using the Raman assisted OTDR. Thanks to the Raman pump, a damaged FC/PC connector can be detected and localised (when printing the "Raman on" and "Raman off" are partly cut. 


\section{CONCLUSIONS}

A technique allowing to increase the dynamic of OTDR for the monitoring of point to multipoint PONs has been demonstrated. The technique is based on Raman amplification of OTDR pulses and is compatible with a centralised approach for the monitoring of PONs. When implemented on a 1:16 PON, it showed a $16 \mathrm{~dB}$ return gain in the dynamic range of the OTDR, which enabled us to detect and localise in the branches faults that could not be seen before.

\section{ACKNOWLEDGEMENTS}

The authors acknowledge support from the FP6 ePhoton/One+ European project and the EUROPA (First/DGTRE) project.

\section{REFERENCES}

[1] "Europe at the speed of light", Proceedings of the FTTH Council Conference, http://www.europeftthcouncil.com/, Barcelona (2007).

[2] F. Caviglia, et al., "OTDR analysis of tree-structured optical networks (splitter PON)", Proc. of the $12^{\text {th }}$ Annual Conference on European Fibre Optic Communications and Networks, EFOC\&N’94, Heidelberg, pp. 188-191 (1994).

[3] H. Schmuck, et al., "Embedded OTDR techniques for cost-efficient fibre monitoring in optical access networks", Proc. of the $32^{\text {th }}$ European Conference on Optical Communications, ECOC'06, Cannes, Mo3.5.4 (2006).

[4] K. Ozawa, et al., "Field trial of in-service individual line monitoring of PONs using a tuneable OTDR", Proc. of the $14^{\text {th }}$ Conference on Optical Fibre Sensors, OFS'00, Venice, pp. 880-883 (2000).

[5] Y. Enomoto, et al., "Over $31.5 \mathrm{~dB}$ dynamic range optical fiber testing system with optical fiber fault isolation function for 32-branched PON", Proc. of Optical Fiber Communications Conference, OFC'03, Atlanta, ThAA3 (2003).

[6] K. Yuksel, et al., "OTDR-based monitoring method for passive optical networks", Proc. of the $8^{\text {th }}$ European Conference on Networks and Optical Communications, NOC'03, Vienna, pp. 255-261 (2003).

[7] K. Yuksel, et al., "OTDR-based fault surveillance method for passive tree-structured networks", Proc. of the 15th International Symposium on Services and Local Access (ISSLS 2004), Edinburg, (2004).

[8] K. Yuksel, et al., "FTTx automated test solution: requirements and experimental implementation", Elect. Letters ,vol. 41, no. 9, pp. 546-547, (2005).

[9] G. Ravet, et al., "Measurement of the distributed Raman gain spectrum is single-mode optical fibres", Proc. of IEEE Laser and Electro-Optics Society Symposium, LEOS 2002, Amsterdam, pp. 242-245 (2002). 